\title{
Cephalochordata do Estuário do Rio Paripe, Itamaracá, Pernambuco.
}

\author{
MARCos SOUTO ALVES ${ }^{1}$ \\ ANA CAROLINA B. LINS-E-SILVA ${ }^{2}$ \\ Luiz AugustinHo MENEZES DA SILVA ${ }^{3}$ \\ LEONARdo CÉSAR DE OliVEIRA MELO
}

1. Departamento de Biologia/ Área de Zoologia - UFRPE

2. Departamento de Biologia/ Área de Ecologia - UFRPE

3. Faculdade de Formação de Professores da Mata Sul/ FAMASUL

\begin{abstract}
RESUMO
São apresentados os resultados dos estudos sobre a taxonomia e bioecologia dos Cephalochordata procedentes de coletas diurnas e noturnas no estuário do Rio Paripe na Ilha de Itamaracá, PE - Brasil ( $7^{\circ} 48^{\prime} \mathrm{S}$ e $34^{\circ} 51^{\prime} \mathrm{W}$ ), no período de janeiro a agosto de 1994 . Registra-se pela primeira vez para a costa do Estado de Pernambuco a espécie Branchiostoma platae Hubbs, 1922, única espécie identificada no presente estudo. A biometria dos indivíduos indicou um comprimento médio de $38.5 \mathrm{~mm}$ e peso médio de $0.145 \mathrm{~g}$. Indivíduos maiores e com maior desenvolvimento gonadal foram observados no período seco, enquanto que o maior número de formas jovens foi encontrado no período chuvoso. Branchiostoma platae é filtrador de microalgas, principalmente diatomáceas. A espécie habita sedimento de granulometria média a grosseira, com acentuada fração de artículos de Halimeda. A salinidade da água apresentou média de 17,7\%o e 4,2ml/L de oxigênio dissolvido.
\end{abstract}

Palavras chave: Cephalochordata, Branchiostoma platae, anfioxo, Rio Paripe.

\section{Abstract}

\section{Cephalochordata of the Paripe river estuary, Itamaracá, Pernambuco.}

Results are given on the taxonomy and ecology of the Cephalochordata obtained from diurnal and nocturnal samples on the Paripe River Estuary, Island of Itamaracá, PE - Brasil ( $7^{\circ} 48^{\prime}$ S and $34^{\circ} 51^{\prime} \mathrm{W}$ ), between January and August 1994. The species Branchiostoma platae Hubbs 1922, the only species recorded on this study, is registered for the first time on the coast of Pernambuco. Biometric measurements revealed a mean length of $38.5 \mathrm{~mm}$ and mean weight of 0.145 g. During the dry season (January to March) individuals with greater gonadal development were observed, whereas greater number of juveniles was recorded during the rainy season (April to July). Branchiostoma platae feeds on microalgae, mainly diatoms. The species lives on sediment type characterised as been coarse to median grained, with many fragments of the algae Halimeda. Mean water salinity was $17.7 \%$ and $4.2 \mathrm{ml}$.l of dissolved oxigen. None individual was collected on plancton samples.

Key words: Cephalochordata, Branchiostoma platae, anfioxus, river.

Tropical Oceanography, Recife: v. 29, n. 2, p. 129-138, 2001. 


\section{INTRODUÇÃO}

O Subfilo Cephalochordata compreende invertebrados marinhos de forma muito semelhante a pequenos peixes, genericamente conhecidos como anfioxos devido ao aspecto afilado, em forma de ponta de lança, observado nas duas extremidades do corpo.

O anfioxo é de grande interesse zoológico porque exibe, em todo o seu ciclo de vida, as três características básicas e distintivas do Filo Chordata - notocorda, fendas branquiais faríngeas e tubo nervoso dorsal. A presença de uma notocorda com bainha extracelular espessa define os cefalocordados e os vertebrados como sendo mais intimamente relacionados entre si do que com os urocordados e reforça a teoria que estes dois grupos tiveram um ancestral comum. A notocorda muscular dos cefalocordados e uma notocorda composta por fibras intracelulares nos vertebrados representam estruturas homólogas que definem cada um desses grupo (Ruppert e Barnes, 1994).

Os Cephalochordata reúnem representantes de três gêneros e pouco mais de vinte espécies. No Brasil, existem referências a estes animais desde o século passado, tendo sido registradas quatro espécies no país: Branchiostoma caribeum Sundevall, 1853 (Sawaya, 1964; Silva, 1977); Branchiostoma platae Hubbs, 1922 (Sawaya e Carvalho, 1938; Sawaya e Carvalho, 1950; Moure, Björnberg et al, 1954; Tommasi, Valente et al, 1972) e Branchiostoma marambaiensis Silva, 1977 (Silva, 1977), encontradas nos estados da região sudeste e sul, além de Amphioxides pelagicus (Günther, 1889) (Björnberg, 1954) coletado no plâncton da Ilha de Fernando de Noronha.

Os anfioxos habitam águas marinhas rasas e limpas em áreas costeiras tropicais e temperadas, onde as formas adultas vivem, na maior parte do tempo, enterradas na areia com a parte anterior do corpo exposta. Por esta particularidade, é comum na literatura especializada a referência a "fundos de anfioxos" ou "areias de anfioxos", quando muitos desses organismos são capturados em dragagens para análises de sedimentos (Kempf, 1967/69; Costa, 1971; Tommasi, Valente et al, 1972), onde são encontrados em diversas profundidades, desde bancos de areia emersos nas baixamares até a profundidade de metros. As larvas de Branchiostoma são um conhecido componente do plâncton do mundo e, em certos mares, ocorrem em elevado número e constituem a maior parte do zooplâncton (Boschung e Shaw, 1988).

Os cefalocordados são importantes como alimento em partes da Ásia, onde são consumidos frescos, fritos ou desidratados. Há registros de uma densidade populacional de cerca de cinco mil indivíduos por metro quadrado de B. caribeum na Baía de Discovery, Jamaica (Ruppert e Barnes, 1994). Apesar de haver registro de exploração econômica, a importância dos anfioxos adquire maiores proporções para a investigação zoológica do que para a economia (Bigelow e Farfante, 1948).

Este trabalho teve como objetivo o estudo taxonômico e bioecológico dos anfioxos da Ilha de Itamaracá, como forma de ampliar os conhecimentos científicos sobre o grupo no estado de Pernambuco.

\section{MATERiAl e Métodos}

As coletas foram coletas, diurnas e noturnas, no estuário do Rio Paripe, Ilha de Itamaracá, PE (Figura 1), no período compreendido entre janeiro e agosto de 1994. Em cada coleta, todo o sedimento contido em uma área demarcada de $50 \times 50 \mathrm{~cm}$, até a profundidade de $10 \mathrm{~cm}$, era coletado e, posteriormente, os indivíduos separados manualmente do sedimento utilizando-se uma peneira com 3mm de abertura de malha. Uma parte do material foi imediatamente fixado em formol a $4 \%$ para análise do conteúdo intestinal, a outra parte foi conservada em água do mar e transferida ao laboratório para estudos morfométricos e, em seguida, conservados em álcool a 70\%. Foram realizados arrastos de plâncton, nos períodos diurnos noturnos, utilizando-se redes com $285 \mu \mathrm{m}$ de

Tropical Oceanography, Recife: v. 29, n. 2, p. 129-138, 2001. 
abertura de malha. Amostras de sedimento foram recolhidas para análises sedimentológicas e de água para estudos hidrológicos. Os anfioxos foram identificados com base no trabalho de Bigelow e Farfante (1948). A biometria incluiu medidas de peso e comprimento total, do rostro ao atrióporo, do atrióporo ao ânus, do ânus a cauda, altura e largura. Foi realizada análise de regressão linear entre o comprimento total e o peso.

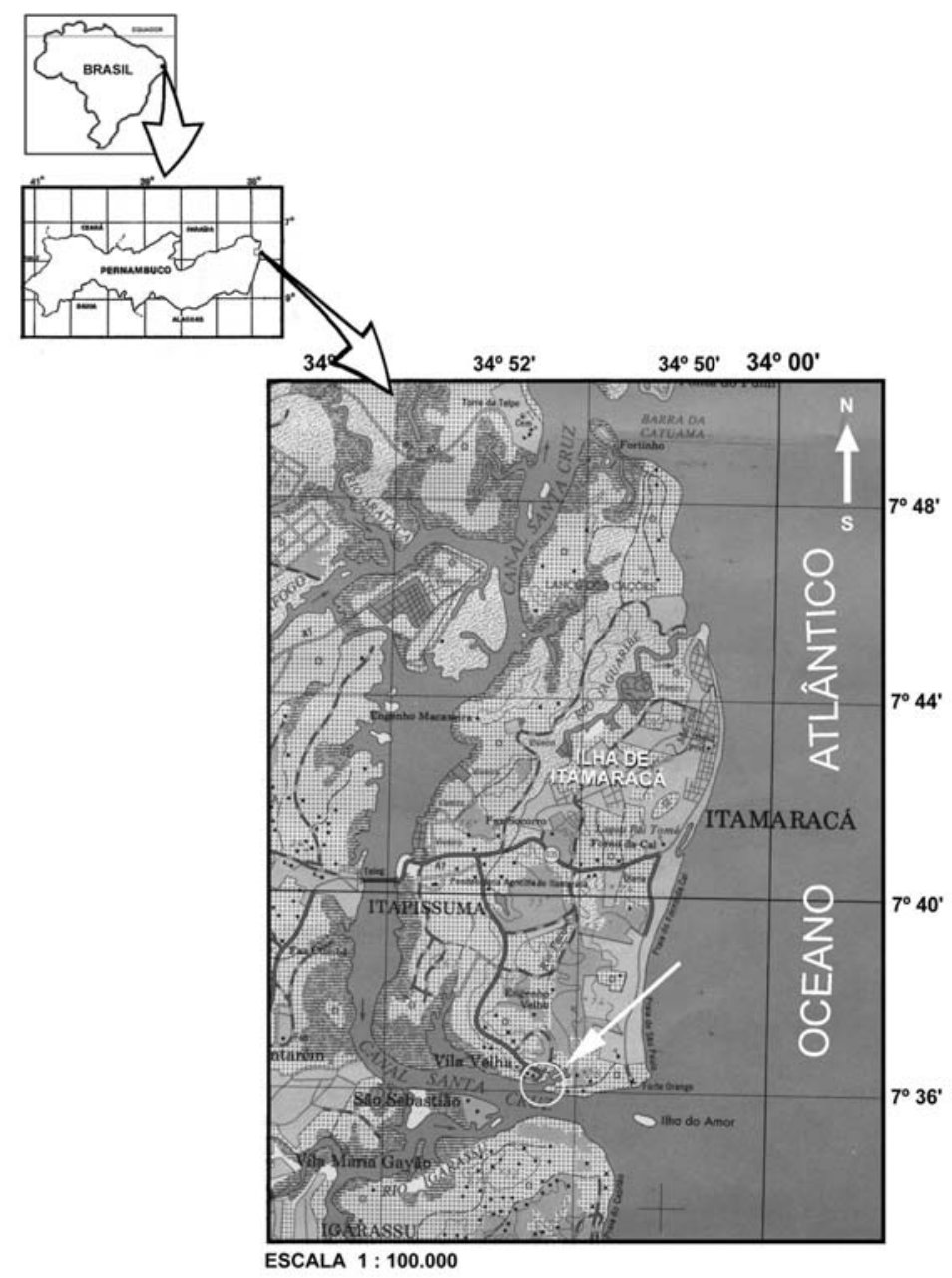

Figura 1 - Localização da área de estudo, estuário do rio Paripe, Ilha de Itamaracá/ PE.

\section{RESUlTADOS E DiscusSÃo}

A identificação do material revelou a presença de apenas uma espécie: Branchiostoma platae Hubbs, 1922. Embora alguns autores tenham registrado a ocorrência de Cephalochordata ou anfioxos em seus trabalhos (CONDEPE, 1983; Neumann-Leitão, Gusmão et al, 1998; Paranaguá, Neumann-Leitão et al, 1999),

Tropical Oceanography, Recife: v. 29, n. 2, p. 129-138, 2001. 
nenhum identificou em nível específico, sendo este o primeiro registro para a espécie na costa de Pernambuco.

Sinopse sistemática:

Filo Chordata

Subfilo Cephalochordata

Classe Leptocardii

Ordem Amphioxi

Família Branchiostomidae

Gênero Branchiostoma Costa, 1834

Espécie Branchiostoma platae Hubbs, 1992.

Referência: Bigelow e Farfante (1948) p. 16-18.

Material examinado: 148 indivíduos, todos procedentes do Estuário do Rio Paripe, Ilha de Itamaracá, Pernambuco, Brasil ( $7^{\circ} 48^{\prime} \mathrm{S}$ e $\left.34^{\circ} 51^{\prime} \mathrm{W}\right)$.

Distribuição geográfica: Foz do Rio La Plata, Sul e Sudeste do Brasil (Bigelow e Farfante, 1948); Rio Grande, RS, Canal de São Sebastião, Canal dos Porcos Grande e Ilha Rapada, Ubatuba, SP (Costa, 1971), Santos, SP (Sawaya e Carvalho, 1938), Baía de Paranaguá, PR (Moure, Björnberg et al, 1954).

Caracteres distintivos: Nadadeira caudal fina e lanceolada. Lobos inferior e superior da nadadeira caudal assimétricos; lobo inferior originando-se em posição anterior ao ponto de origem do lobo superior (Figura 2). Ânus situado consideravelmente anterior ao ponto médio do lobo inferior da nadadeira caudal. Altura da nadadeira dorsal equivalente a cerca de1/4 da altura do corpo. Câmaras da nadadeira dorsal mais altas que largas variando em número de 280 a 330 (Figura $3)$.

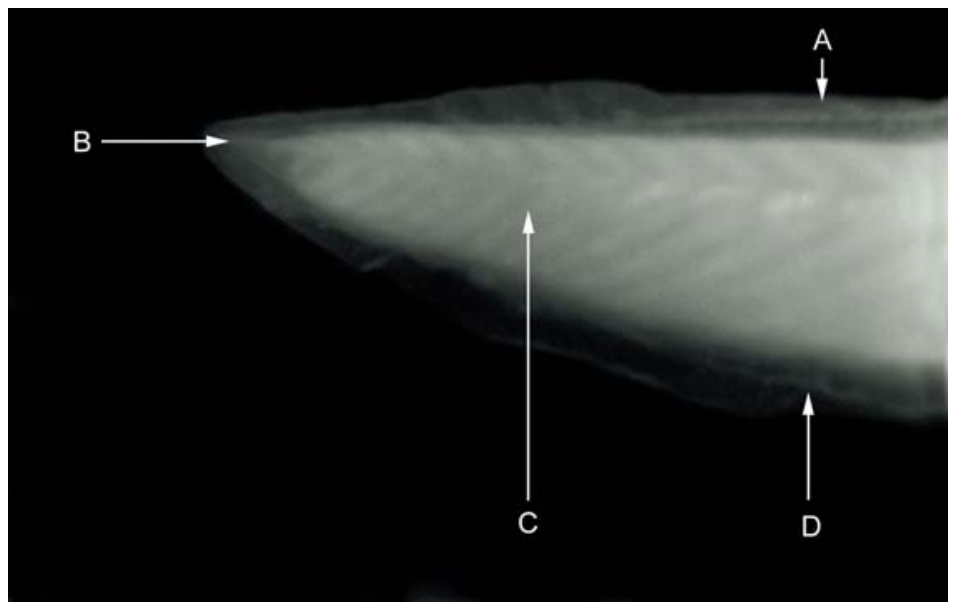

Figura 2.- Extremidade posterior de Branchiostoma platae Hubbs, 1922, coletado no Rio Paripe, Ilha de Itamaracá - PE. (A) lobo superior da nadadeira caudal, (B) extremidade posterior da nadadeira caudal, (C) miômeros, (D) lobo inferior da nadadeira caudal.

Tropical Oceanography, Recife: v. 29, n. 2, p. 129-138, 2001. 


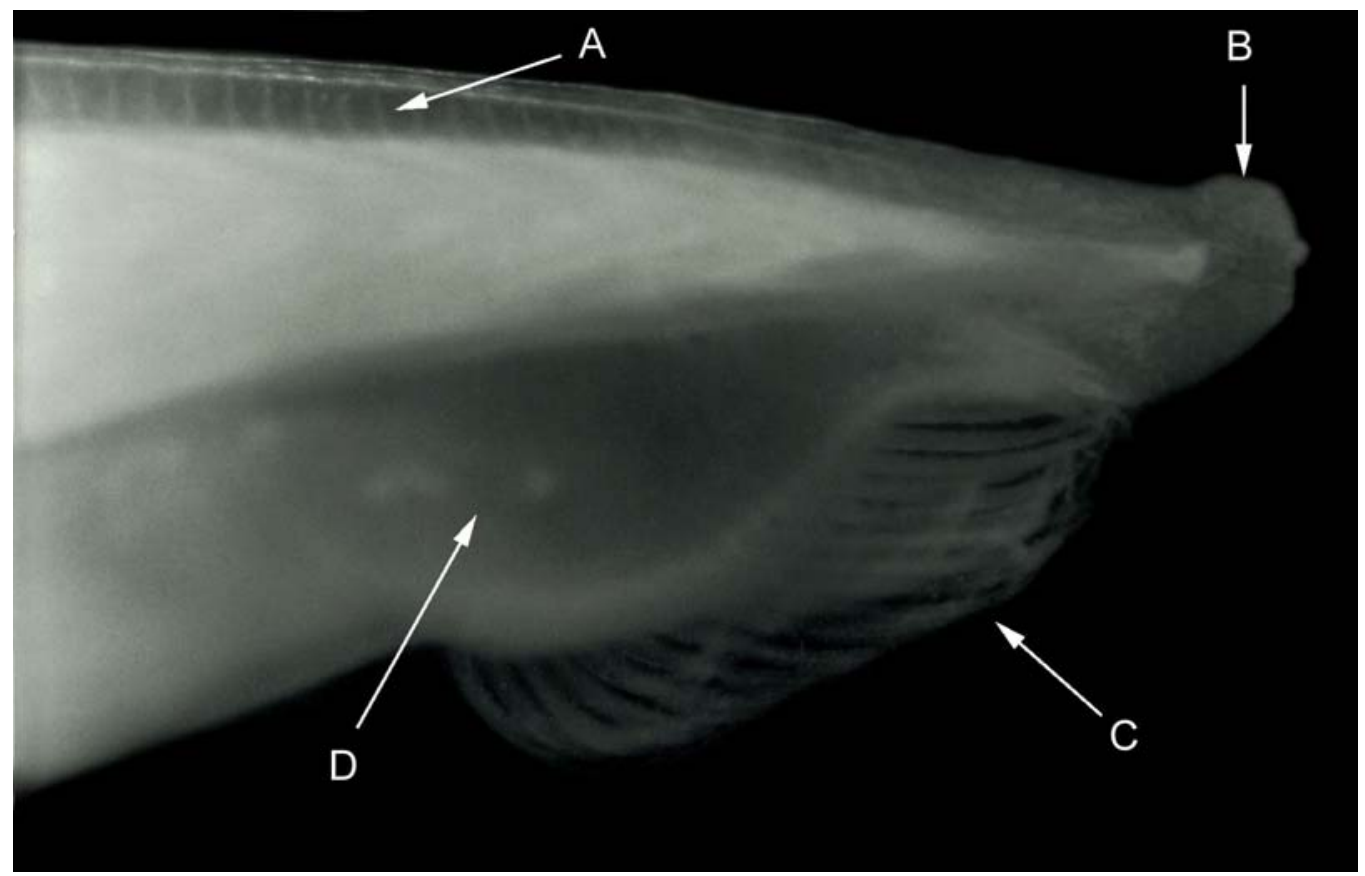

Figura 3 - Extremidade anterior de Branchiostoma platae Hubbs, 1922, coletado no Rio Paripe, Ilha de Itamaracá - PE. (A) câmaras da nadadeira dorsal, (B) rostro, (C) cirros bucais, (D) vestíbulo.

Dos 148 indivíduos examinados, 58 foram coletados no período diurno e 90 no período noturno, todos procedentes do sedimento, não havendo registro do animal nos arrastos de plâncton, diurnos ou noturnos. Os cefalocordados, reconhecidamente bentônicos endopsâmicos de vida livre, também podem nadar ativamente na coluna d'água, por meio de contrações da musculatura lateral do corpo, compondo o zooplâncton, principalmente durante a noite. Boschung e Shaw (1988) registraram a ocorrência de adultos de Branchiostoma spp no plâncton da Louisiana, discutindo a possibilidade de haver migração vertical relativa a fatores morfométricos e ecológicos. Em nosso trabalho não foi coletado nenhum indivíduo em arrastos de plâncton.

A maior densidade populacional ocorreu no mês de agosto (Figura 4), quando foi observada predominância de formas jovens, sendo aquelas que apresentaram menor média de peso (Figura 5), enquanto que os indivíduos com menor comprimento total foram coletados no mês de junho, o que representa a estação chuvosa no local de coleta (Figura 6). Para Branchiostoma floridae, Stokes (1996) encontrou que a estação reprodutiva pode durar até 5 meses, os animais apresentam crescimento no verão, praticamente cessando no inverno. Indivíduos maiores e com maior desenvolvimento gonadal (Figura 7) foram observados nos meses de janeiro a março.

O peso médio encontrado foi de 0,067g, com mínimo de 0,003g e máximo de 0,2498g, enquanto o comprimento variou de 7 a $46 \mathrm{~mm}$, com média de $27,47 \mathrm{~mm}$. A análise de correlação e regressão linear entre o comprimento total e o peso total mostrou haver correlação linear positiva e de forte intensidade $(r=0,8411, p \leq 0,05)$ entre as duas variáveis (Figura 8).

A salinidade da água apresentou média de 17,7\%o durante os horários de coleta, sempre nas baixa-mares, o que se enquadra nos valores mais baixos de salinidade observados por Macêdo, Montes et. al (1998), nas desembocaduras do rios que desaguam no Canal de Santa Cruz. Para o gênero Branchiostoma, Boschung e Shaw (1988) destacam que valores letais de salinidade máxima e mínima variam de acordo com a espécie, o estágio do desenvolvimento e a duração da exposição.

Tropical Oceanography, Recife: v. 29, n. 2, p. 129-138, 2001. 
Em relação ao oxigênio, Boschung e Shaw (1988) registraram a ocorrência de Branchiostoma em águas com taxas variáveis entre 5.04 a $9.28 \mathrm{mg} / \mathrm{l}$, valores equivalentes aos obtidos no estuário do Rio Paripe, que apresentaram valor médio de 4,2ml/l.

Os cefalocordados demonstram preferência por areias limpas, de granulometria média a grossa, geralmente com muitos fragmentos de origem orgânica, principalmente algas calcárias, conchas de moluscos e carapaças de crustáceos. Segundo Costa (1971) a fração de origem animal está sempre representada de maneira notável e constitui uma característica constante dos biótopos onde se instalam os "fundos à base de anfioxos" em todos os lugares do mundo. O sedimento analisado apresentou granulometria média a grosseira com acentuada fração de artículos de Halimeda. Segundo Kempf (1967/69) esse tipo de sedimento é resultante de areias quartzosas na dependência dos estuários e areia calcária biodetrítica ligada à presença dos recifes.

No estuário do Rio Paripe, os anfioxos estão concentrados num banco de areia emerso nas baixa-mares, que se encontra margeado por lama escura e redutora. Costa (1971) enfatiza a presença dos anfioxos em bancos de sedimento arenoso isento de lama, porém, uniformemente rodeado por ela, e atribui este fato à acentuada ação hidrodinâmica.

Os hábitos alimentares dos anfioxos não são abordados na literatura disponível. Neste trabalho, a análise qualitativa do conteúdo intestinal revelou que a dieta é constituída basicamente por diatomáceas, entre as quais foram identificadas as espécies Navicula humerosa, Navicula sp, Amphora augusta, Grammatophora oceanica, Grammatophora sp, Gyrosigma balticum, Cocconeis sp e Frustulia rhomboides. Segundo Eskinazi-Leça, Macêdo et al (1980) a presença de espécies litorais, bentônicas e epífitas, no estuário do Rio Paripe deve-se, sobretudo, a baixa profundidade e aos prados de fanerógamas marinhas situados em áreas adjacentes. Com exceção de Frustulia, todos os gêneros identificados são citados, também, como constituintes da dieta alimentar de peixes da Família Mugilidae, tais como, Mugil liza (tainha) e Mugil curema (curimã), cujo hábito alimentar iliófago indica a presença de tais diatomáceas no sedimento ou nas águas circundantes (EskinaziLeça, Gonçalves da Silva et al, 1985/86).

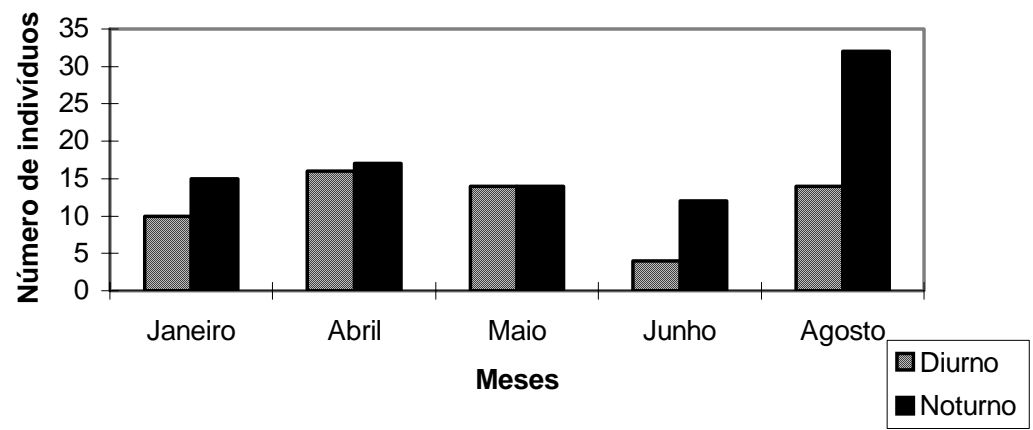

Figura 04 - Freqüência de ocorrência mensal de Branchiostoma platae Hubbs, 1922 no estuário do Rio Paripe - Ilha de Itamaracá/PE.

Tropical Oceanography, Recife: v. 29, n. 2, p. 129-138, 2001. 


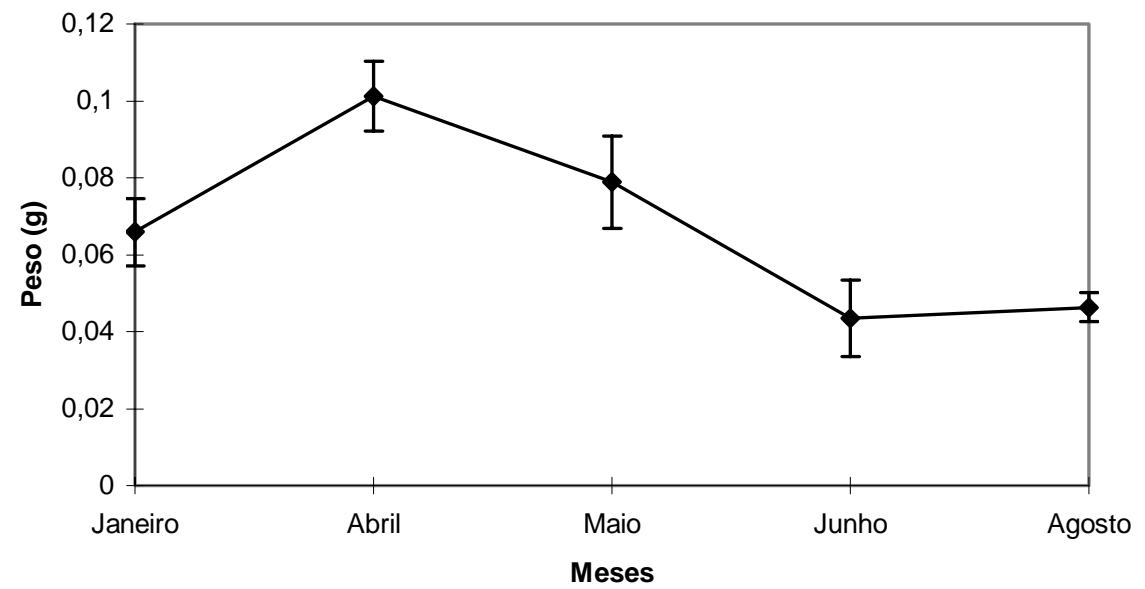

Figura 05 - Variação mensal no peso em Branchiostoma platae Hubbs, 1922 do estuário do Rio Paripe - Ilha de Itamaracá/PE.

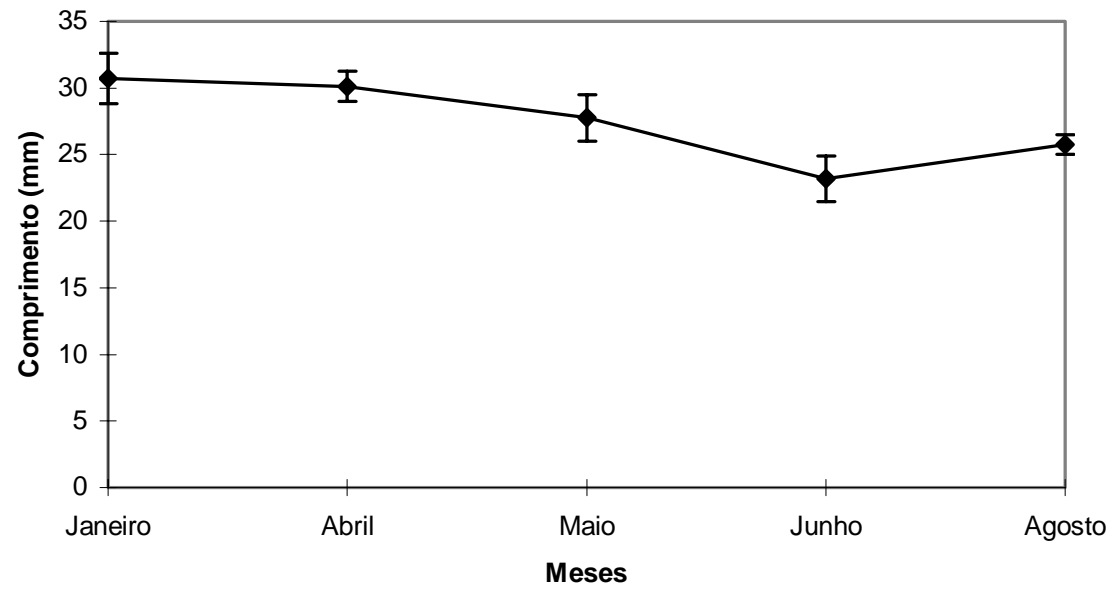

Figura 06 - Variação mensal no comprimento total em Branchiostoma platae Hubbs, 1922 do estuário do Rio Paripe - Ilha de Itamaracá/PE. 


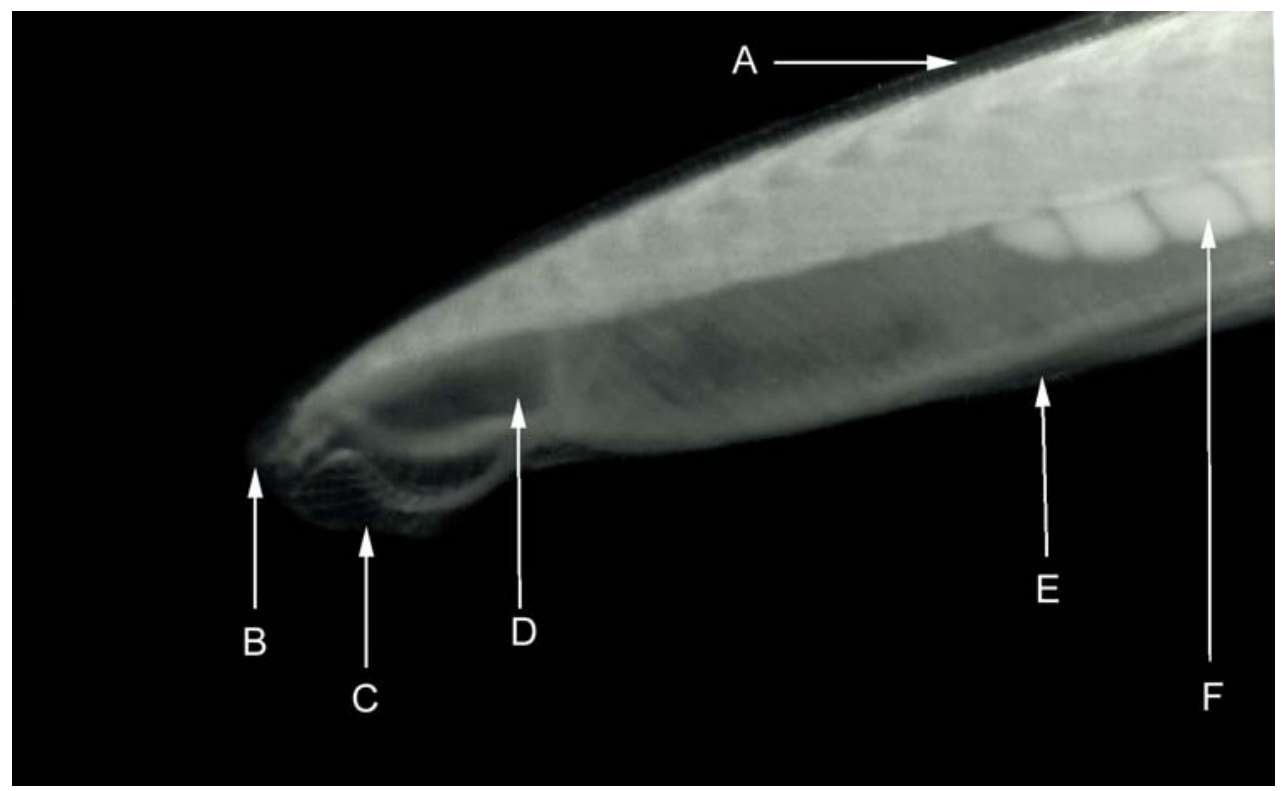

Figura 7 - Detalhe da extremidade anterior de Branchisotoma platae Hubbs, 1922, coletado no Rio Paripe, Ilha de Itamaracá - PE. (A) nadadeira dorsal, (B) rostro, (C) cirros bucais, (D) vestíbulo, (E) pregas metapleurais e (F) gônadas.

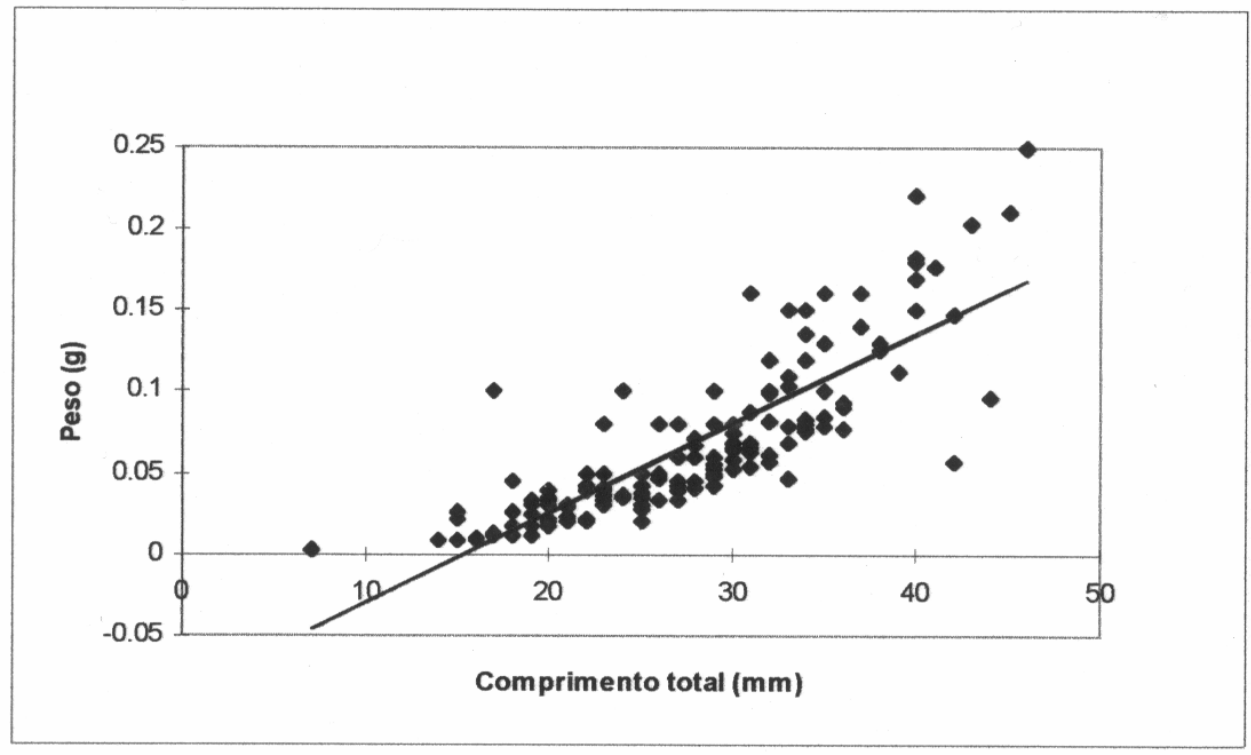

Figura 08 - Regressão linear entre o comprimento total e o peso de Branchiostoma platae Hubbs, 19: do estuário do Rio Paripe Itamaracá/PE. A equação da reta foi y=0,0055x - 0,0844.

Tropical Oceanography, Recife: v. 29, n. 2, p. 129-138, 2001. 


\section{AgRADECIMENTOS}

Os autores são gratos à Dra. Maryse Nogueira Paranaguá, pelo apoio no desenvolvimento desta pesquisa, pela leitura e valiosas sugestões no manuscrito.

\section{REFERÊNCIAS BIBLIOGRÁFICAS}

BIGELOW H. B.; FARFANTE, I. P. Lancelets. In Fishes of the Western North Atlantic. Memoir Seaves Foundation Marine Research. New Haven, v.1, n.17, p.1-28, 1948.

BJÖRNBERG, T. K. S. Occurrence of Amphioxides pelagicus (Gunther) in Fernando de Noronha Is. Plankton. Boletim do Instituto Oceanográfico. São Paulo, v. 5 p. 83-86, 1954.

BOSCHUNG, H. T.; SHAW, R. F. Occurrence of planktonic lancelets from Louisiana's continental shelf, with a review of pelagic Branchiostoma (Order Amphioxi). Bulletin of Marine Science. Miami, v. 43, n. 2, p. 229-240, 1988.

CONDEPE Síntese Ecológica. In: Caracterização do complexo estuarino-lagunar da Área de Suape (Pernambuco - Brasil). Recife: CONDEPE. 1983, 107p. v. 1.

COSTA, H. R. da. Aspectos das biocenoses à base de "anfioxus” no Brasil. Arquivos do Museu Nacional. Rio de Janeiro, v. 54, p.85-89, 1971.

ESKINAZI-LEÇA, E., GONÇALVES DA SILVA, M. da G; VASCONCELOS FILHO, A. de L.. Diatomáceas do conteúdo estomacal de Mugil curema Valenciennes,1836 e Mugil liza Valenciennes, 1836 (Pisces Mugilidae). Trabalhos Oceanográficos Universidade Federal de Pernambuco. v. 19, p. 131-146, 1985/86.

ESKINAZI-LEÇA, E., MACÊDO, S. J. de; PASSAVANTE, J. Z. de O. Estudo ecológico da região de Itamaracá, Pernambuco, Brasil. V Composição e distribuição do microfitoplâncton na região do Canal de Santa Cruz. Trabalhos Oceanográficos Universidade Federal de Pernambuco. v. 15, p. 185-262, 1980.

KEMPF, M. Nota preliminar sobre os fundos costeiros da Região de Itamaracá (Norte do Estado de Pernambuco, Brasil).Trabalhos Oceanográficos Universidade Federal de Pernambuco. Recife. v. 9/11, p. 95-100, 1967/69.

MACÊDO, S. J. de; MONTES, M. de J. F. Características abióticas da área. In: GERENCIAMENTO AMBIENTAL PARTICIPATIVO: APLICAÇÃO AO CASO DOS MANGUEZAIS DO CANAL DE SANTA CRUZ-PE. Recife: FUNEP/FUNDAJ/UFPE/UFRPE. 1988.

MOURE, J. S.; BJORNBERG, T. K. S. et al. Protochordata ocorrentes na entrada da Baía de Paranaguá. Dusenia, v..5/6, p.233-242, 1954.

NEUMANN-LEITÃO, S.; GUSMÃO, L. M. de; et al. Biodiversidade do mesozooplancton do Nordeste do Brasil. Trabalhos Oceanográficos Universidade Federal de Pernambuco. Recife. v.26, n. 1, p.27-34, 1999.

Tropical Oceanography, Recife: v. 29, n. 2, p. 129-138, 2001. 
PARANAGUÁ, M. N.; NEUMANN-LEITÃO, S. et al. Management in north-eastern Brazil: faunal biodiversity in ecosystems and sustainable development II. Advances in Ecological Sciences. Eds. C. A. Brebbia and J. L. Usó. Wit Press. Southampton, Boston. 1999, p. 57-68.

RUPPERT, E. E. e BARNES, R. D. Invertebrate Zoology. Saunders College Publishing. Orlando, 1953, 1056p.

SAWAYA, P. Acrania (Protocordados) in VANZOLINI, P. E., 1964. ed. História Natural dos Organismos Aquáticos do Brasil. São Paulo, FAPESP. 1964, p. 305-308.

SAWAYA, P. e CARVALHO, P. J. Ocorrência de Branchiostoma (Anfioxus) na Baía de Santos. Bolm. Biol. Fac. Med. (N. S.). São Paulo, v. 3 n. 2, p. 43-46, 1938.

SAWAYA, P. e CARVALHO, P. J. On the Branchiostoma (Amphioxus) of the coast of São Paulo. Boletim da Faculdade de Filosofia e Ciências da Universidade de São Paulo. v. 15, p 235-237, 1950.

SILVA, S. H. G. da. Anfioxos da Marambaia: Aspectos taxonômicos e ecológicos. Rio de Janeiro. Dissertação (Mestrado) Universidade Federal do Rio de Janeiro. 1977, 84p.

TOMMASI, L. R.; VALENTE, M.T.M. et al. Cephalochordata da Região de Ilha Grande (RJ). Boletim do Instituto Oceanográfico. São Paulo, Instituto Oceanografico da USP, n. 21, p. 149162, 1972. 